\title{
Economic Games Quantify Diminished Sense of Guilt in Patients with Damage to the Prefrontal Cortex
}

\author{
Ian Krajbich, ${ }^{1}$ Ralph Adolphs, ${ }^{1,2}$ Daniel Tranel, ${ }^{2}$ Natalie L. Denburg, ${ }^{2}$ and Colin F. Camerer ${ }^{1}$ \\ ${ }^{1}$ Division of the Humanities and Social Sciences, California Institute of Technology, Pasadena, California 91125, and ${ }^{2}$ Department of Neurology, The \\ University of Iowa, Iowa City, Iowa 52242
}

\begin{abstract}
Damage to the ventromedial prefrontal cortex (VMPFC) impairs concern for other people, as reflected in the dysfunctional real-life social behavior of patients with such damage, as well as their abnormal performances on tasks ranging from moral judgment to economic games. Despite these convergent data, we lack a formal model of how, and to what degree, VMPFC lesions affect an individual's social decision-making. Here we provide a quantification of these effects using a formal economic model of choice that incorporates terms for the disutility of unequal payoffs, with parameters that index behaviors normally evoked by guilt and envy. Six patients with focal VMPFC lesions participated in a battery of economic games that measured concern about payoffs to themselves and to others: dictator, ultimatum, and trust games. We analyzed each task individually, but also derived estimates of the guilt and envy parameters from aggregate behavior across all of the tasks. Compared with control subjects, the patients donated significantly less and were less trustworthy, and overall our model found a significant insensitivity to guilt. Despite these abnormalities, the patients had normal expectations about what other people would do, and they also did not simply generate behavior that was more noisy. Instead, the findings argue for a specific insensitivity to guilt, an abnormality that we suggest characterizes a key contribution made by the VMPFC to social behavior.
\end{abstract}

Key words: prefrontal; lesion; social preference; guilt; neuroeconomics; ventromedial

\section{Introduction}

How people anticipate and react to the fortunes of others is a central issue in all social sciences, but its psychological and neurobiological underpinnings are not well understood. Experimental economists and psychologists have studied how people value monetary payoffs (gains or losses) to others, using simple economic games designed to measure deviations from pure selfinterest in monetary terms. The results of these studies have led to mathematical descriptions of "social preference": models that describe how people integrate the monetary outcomes they themselves receive, with those of other players (Fehr and Schmidt, 2001; Camerer and Fehr, 2006).

A parallel line of research in patients with focal brain lesions has described qualitative patterns of altered real-life social behavior, and made inferences about the impaired social emotions that underlie it. Damage to the ventromedial prefrontal cortex

Received 0ct. 21, 2008; revised Dec. 22, 2008; accepted Jan. 8, 2009.

This work was supported by a program project grant to A. R. Damasio from the National Institute of Neurological Disorders and Stroke and by grants from the National Institute of Mental Health (R.A.), the National Institute on Drug Abuse (D.T.), the National Science Foundation (R.A. and C.F.C.), and the Gordon and Betty Moore Foundation (R.A. and C.F.C.). Hanna Damasio and Jessica Wisnowski (Dornsife Cognitive Neuroscience Imaging Center, University of Southern California) conducted the neuroanatomical analysis of the patients and provided the illustration of the VMPFC group. We thank all participants for their participation in the experiments, Amanda Hornaday, Kodi Scheer, and Ruth Henson for help with scheduling and testing the subjects, Ming Hsu for suggestions with analysis and comments on this manuscript, Michael Koenigs for comments on this manuscript, and Jonathan Leland for general comments.

The authors declare no competing financial interests.

Correspondence should be addressed to Ralph Adolphs, Division of the Humanities and Social Sciences, California Institute of Technology, Pasadena, CA 91125. E-mail: radolphs@caltech.edu.

D01:10.1523/JNEUROSCI.5086-08.2009

Copyright $\odot 2009$ Society for Neuroscience $\quad$ 0270-6474/09/292188-05\$15.00/0
(VMPFC), in particular, is known to impair real-world social functioning, decision-making, and planning (Damasio, 1994; Anderson et al., 1999, 2006; Bechara et al., 2000; Beer et al., 2003), consistent with studies that have implicated the VMPFC in processing emotional value attributed to rewards and punishments (Fuster, 1989; Bechara et al., 1997; Rogers et al., 1999; Breiter et al., 2001; Critchley et al., 2001; Kringelbach, 2005).

Whereas some of these studies have investigated impairments in decision-making, the research on the abnormal social behaviors exhibited by VMPFC patients has been largely based on qualitative observation, interviews with family members, or nonchoice data (Ackerly and Benton, 1947; Damasio, 1994; Anderson et al., 2006; Shamay-Tsoory et al., 2007). These data and observations suggest the hypothesis that the abnormal social behaviors following damage to the VMPFC may arise, at least in part, from impairments to social emotions, particularly guilt and envy (Shin et al., 2000; Damasio, 2003; Koenigs and Tranel, 2007; Koenigs et al., 2007). However, none of the previous studies have used formal choice models to isolate and quantify behaviors that would be elicited by these social emotions. Doing so would help provide a general model of how VMPFC patients behave in social tasks and help to distinguish between several competing hypotheses about the roles of VMPFC in social behavior.

To address this hypothesis, we evaluated VMPFC lesion patients (Fig. 1) and comparison subjects on three types of games that are commonly used to measure social preferences in experimental economics: dictator, ultimatum and trust games (Camerer, 2003). We used a stochastic model of choice from economics that includes numerical measures of envy $(\alpha)$ and guilt $(\beta)$, defined as the relative weights on the difference in earnings when 

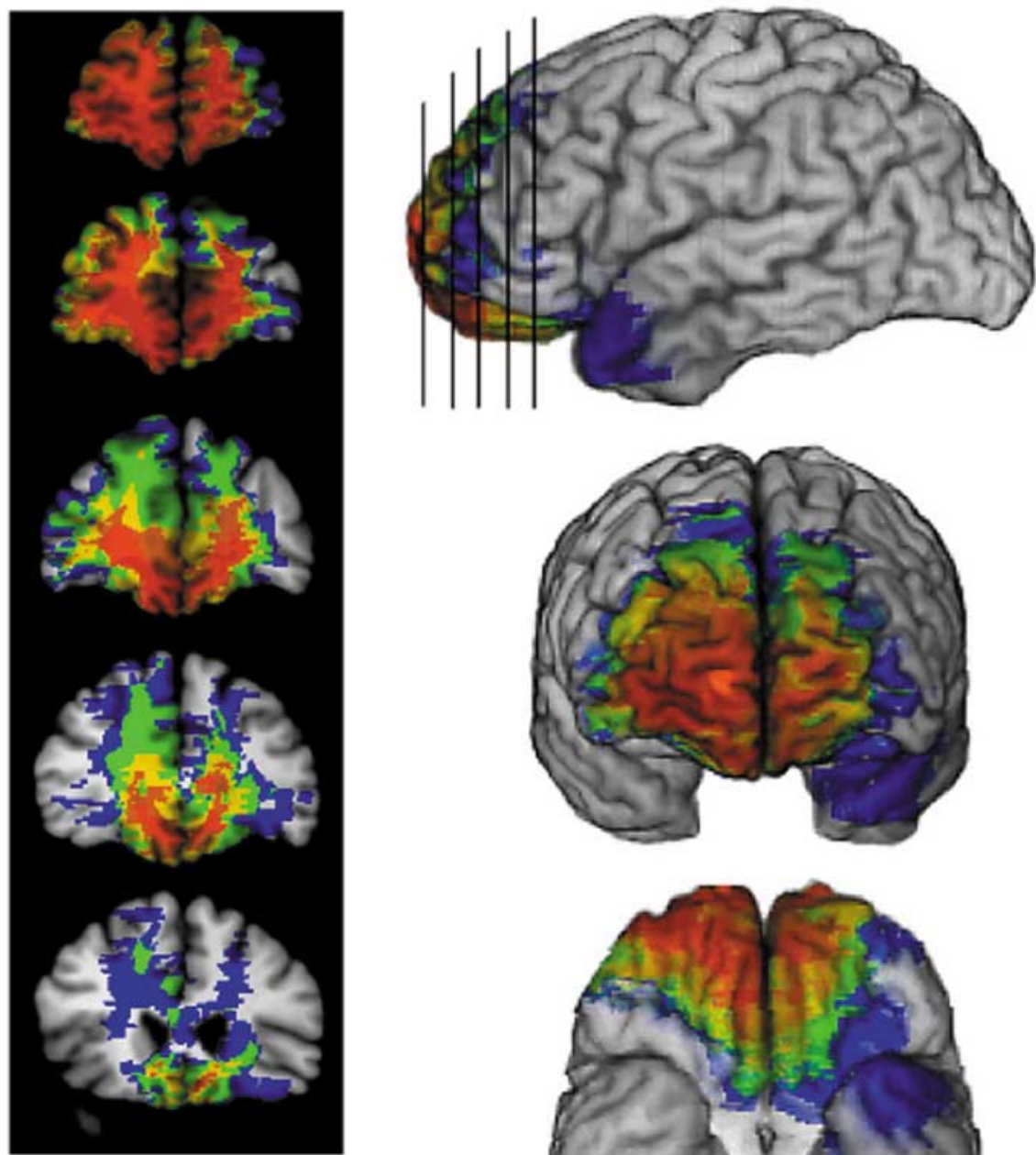

Materials and Methods

Participants. We tested six patients with focal bilateral damage to the ventromedial prefrontal cortex, encompassing both anterior and posterior sectors (Fig. 1), who had known impairments in real-life social behavior, and in particular in social emotions (Damasio, 2003; Koenigs et al., 2007), but were otherwise comparable in age, education, IQ and basic cognitive functioning to the comparison groups. Our VMPFC patients, as well as 20 brain-damaged comparisons with focal, stable lesions of the brain (BDC; with damage in diverse cortical regions but excluding the VMPFC) were recruited from the Cognitive Neuroscience Patient Registry at the University of Iowa from a larger sample (in particular, for anatomical specificity, we excluded several potential VMPFC patients because their lesions were too large and encompassed too many other sectors of the prefrontal cortex). A third comparison group consisted of 16 healthy participants (NC) of similar age and IQ (see supplemental Table 1, available at www.jneurosci.org as supplemental material). All six VMPFC patients had participated in previous published studies that examined this region of the brain (Hsu et al., 2005; Koenigs et al., 2007), and had given informed consent to participate in our study.

Procedure. In a one-shot dictator game, each subject was asked to divide 50 points between him or herself and an anonymous stranger. In the context of our economic model, offering $>0$ points is an example of guilt, the preference for giving up some of one's own payoffs to achieve a more equal division. In a one-shot ultimatum game, shares of 50 points were again allocated, but the recipient of the offer could accept it (in which case both players earn their respective shares) or reject it (in which case neither player gets anything). Subjects played the ultimatum game twice, once as the proposer where they were asked how much they would offer, and once as the recipient where they were asked for the minimum amount they would demand. In the context of our economic model, rejecting an offer is an example of envy, the preference for getting nothing (but an equal share) over accepting an uneven division.

We next assessed trust and trustworthiness using binary-choice "trust" games. In these games, the first player (investor) chooses to either End the game, in which case both players earn 20 points, or to Trust. If Trust is chosen, the second player (trustee) chooses to either Repay or Betray. Three versions of the trust game varied the temptation payoff $T=45, T=60$, and $T=100$. A fourth game used $T=60$ and others earn more (envy) or less (guilt) than oneself (Fehr and Schmidt, 1999). Using this model, we were able to analyze the pooled data from all three types of games to infer subject-specific social preference parameters and compare them across groups, expecting to see that the VMPFC lesion patients would exhibit less guilt and more envy than the comparison subjects, based on previous results which have shown that VMPFC lesion patients reject unfair ultimatum offers and behaviorally appear to show substantially diminished guilt (Koenigs and Tranel, 2007; Koenigs et al., 2007). changed the Repay payoffs to $R=(30,30)$. Each subject played all four versions twice, once as the investor and once as the trustee. Variation in the willingness to Trust and Betray for various Repay/Betray payoff combinations allows us to gauge the relative weights one puts on one's own payoffs and on equality. For more details on these games, see supplemental Figure 1 (available at www.jneurosci.org as supplemental material).

All subjects were tested individually at the University of Iowa, and told that they were playing against another person at Caltech with whom the experimenter was in contact over the phone. During the tasks, the experimenter spoke with the other person on the phone and described the 
subject's decisions as well as asked for the other player's decision (except for the dictator game). No other information was provided about the other player (subjects did not actually speak with the other player, nor was any information communicated about the other player's emotional reactions). Subjects were told that in every game, including the four versions of the trust game, they were interacting with a different person. The behavioral patterns we saw in comparison subjects using this procedure were comparable to those in many other experiments in which subjects played with other people for monetary rewards.

All games were played according to a fixed strategy, but this was not known to the subjects. Every subject participated first in the dictator game, then the ultimatum game, and finally the trust games [in fixed order: $T=60, T=45, T=$ 100 , and last $T=60$ with Repay $=(30,30)]$. Subjects played for points throughout and were told that these would be converted to real money at the end of the experiment; they were not told of the conversion formula and every subject was given $\$ 20$ at the end.

Modeling choice and guilt/envy. We used a model of economic choice in which players weigh their own payoffs against the (squared) deviations between their payoffs and what others get (Fehr and Schmidt, 1999; Bolton and Ockenfels, 2000; Charness and Rabin, 2002). The coefficients on the squared deviations are the weights on guilt and envy compared with one's own earnings, depending on whether the deviations are above or below an equal share, respectively. Denote player 1's payoff from a particular game as $x_{1}$ and player 2's payoff as $x_{2}$. Then the model formalizes player 1's overall utility as follows: $u_{1}\left(x_{1}, x_{2}\right)=x_{1}-\alpha\left[\operatorname{Min}\left\{x_{1} /\left(x_{1}+\right.\right.\right.$ $\left.\left.\left.x_{2}\right)-1 / 2,0\right\}\right]^{2}-\beta\left[\operatorname{Max}\left\{x_{1} /\left(x_{1}+x_{2}\right)-1 / 2,0\right\}\right]^{2}$.

The weights $\alpha$ and $\beta$ measure how much people dislike earning an unfair payoff share which deviates from $1 / 2$, relative to earning more money (higher $x_{1}$ ). We consider two cases, one where the deviations are below equality, in which case the relevant parameter is envy $(\alpha)$, and one where the deviations are above equality, in which case the relevant parameter is guilt $(\beta)$. A weight of $\beta=0$ corresponds to pure selfishness with no guilt; a person with $\beta=0$ is indifferent about the other player's payoffs and will simply maximize his own payoffs. A higher $\beta$ corresponds to a greater willingness to sacrifice one's own payoffs to create more equal payoffs for everyone. Similarly, a weight of $\alpha=0$ corresponds to pure self-interested behavior with no envy of other players' payoffs. A high $\alpha$ signifies a greater willingness to sacrifice one's own payoffs just to ensure that others will not earn higher payoffs.

We combined this model of fairness preference with a logistic model of stochastic choice (Luce, 1959; Hsu et al., 2005). In our games, players chose between actions which create two different payoff allocations $X=$ $\left(x_{1}, x_{2}\right)$ and $Y=\left(y_{1}, y_{2}\right)$. The utilities of these allocations are given by the formula above, yielding $u(X)$ and $u(Y)$. We assume choices are imperfectly sensitive to these differences in utility, according to the "softmax"

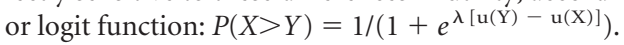

In this "noisy" decision making model, $P(X>Y)$ is the probability that $X$ is chosen over $Y$, and $\lambda$ is a parameter that calibrates how responsive a player's choices are to differences in utility. A $\lambda=0$ corresponds to pure random choice; higher values of $\lambda$ mean subjects are more responsive to utility differences and more often pick the option that yields a higher utility.

The analysis of guilty decision-making was done using the dictator offer, the ultimatum offer and the trustee decisions in the trust games. The analysis of envious decision-making was done using the investor decisions in the trust games. One problem with using the trust and ultimatum games is the confound between a subject's own sense of guilt or envy and his perception of how fair-minded others are. It is therefore necessary to estimate the envy and guilt parameters $\alpha$ and $\beta$, along with expectation parameters for the envy and guilt weights of others, denoted $E(\alpha)$ and $E(\beta)$.

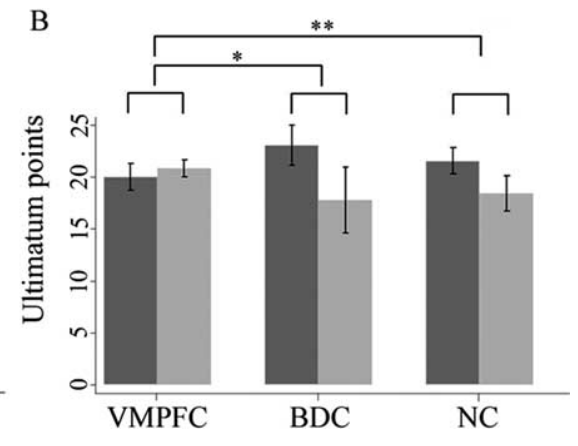

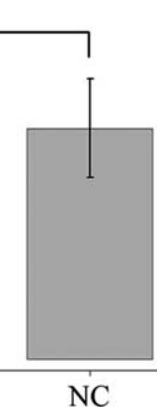
Its on dictator and ultimatum games (with SE bars). $A$, Mean number of points (out of 50 ) given to the
ame. VMPFC lesion patients gave significantly less than the control groups. One NC subject did not Mean number of points (out of 50 ) offered in the proposer role of the ultimatum game (dark bars), and f 50) demanded in the recipient role of the ultimatum game (light bars). We compare the mean matum games.

Parameter estimation was done using maximum likelihood estimation (MLE) (see supplemental Methods, available at www.jneurosci.org as supplemental material). An infeasibly large dataset would be required to estimate all parameters separately for each subject; given our primary interest in the social preference parameters and not the noise parameters, we estimated the two separate $\lambda$ values at the group level (one each for the analyses of the decisions involving guilt, one each for the analyses of the decisions involving envy), and then were able to estimate the other parameters $[\alpha, \beta, E(\alpha)$, and $E(\beta)]$ for each subject individually.

\section{Results}

Figure 2 summarizes the offers and demands made in the dictator and ultimatum games, out of 50 points. The data produced by comparison subjects were comparable to those in many other normal populations (Camerer, 2003). Two patterns were significant: First, VMPFC patients gave less in the dictator games (only 4.7 of 50, compared with 12 and 18 for the comparison groups), consistent with the hypothesis that they would exhibit less guilt. Second, in the ultimatum game we did not see significant differences in the offers or demands, but a striking difference between the groups was that the gap between ultimatum offers and demands was zero for all but one of the VMPFC patients (and only a -5 point difference for that one). In contrast, comparison subjects generally offered more than they demanded [as in most other studies (Camerer, 2003)]. This result is also consistent with our hypothesis about guilt, since the gap between ultimatum offers and demands is an approximate measure for how much more a subject is willing to offer above what he/she believes is the minimum acceptable to the other player.

Table 1 shows the percentage of Trust and Repay choices in the trust games. Looking at the investor behavior, the VMPFC group chose to trust less often than the BDC group in all 4 combinations, and less than the $\mathrm{NC}$ group in 3 of 4 combinations (trusting equally in one case). In the trustee role, the VMPFC group repaid less often than the control groups across the board but also seemed to generally reduce their Repay rates as the incentives to Betray increased.

Because each subject played all four trust games, we were able to combine their choices to form a composite index of trust (the percentage of games in which they chose Trust) and trustworthiness (the percentage of games in which they chose Repay), denoted "Avg" in Table 1. Using this measure, the VMPFC patients were significantly less trustworthy than the NC group. The BDC group was not significantly different from the VMPFC or NC 
Table 1. Percentages (raw proportions) of "Trust" and "Repay" choices in trust games with different temptation values $T$ and different Repay payoffs $R$

\begin{tabular}{|c|c|c|c|c|c|c|c|c|c|c|}
\hline \multirow[b]{2}{*}{ Group $(N)$} & \multicolumn{5}{|c|}{ Investor: percent who chose Trust } & \multicolumn{5}{|c|}{ Trustee: percent who chose Repay } \\
\hline & $T=45$ & $T=60$ & $\begin{array}{l}T=60 \\
R=(30,30)\end{array}$ & $T=100$ & Avg & $T=45$ & $T=60$ & $\begin{array}{l}T=60 \\
R=(30,30)\end{array}$ & $T=100$ & Avg \\
\hline VMPFC (6) & $50(3 / 6)$ & $50(3 / 6)$ & $50(3 / 6)$ & $33(2 / 6)$ & $46(11 / 24)$ & $33(2 / 6)$ & $33(2 / 6)$ & $17(1 / 6)$ & $0(0 / 6)$ & $21(5 / 24)$ \\
\hline $\mathrm{BDC}(17)$ & $59(10 / 17)$ & $65(11 / 17)$ & $53(9 / 17)$ & $71(12 / 17)$ & $62^{0.23}(42 / 68)$ & $47(8 / 17)$ & $59(10 / 17)$ & $35(6 / 17)$ & $24(4 / 17)$ & $41^{0.11}(28 / 68)$ \\
\hline NC (16) & $56(9 / 16)$ & $63(10 / 16)$ & $50(8 / 16)$ & $50(8 / 16)$ & $55^{0.32}(35 / 64)$ & $75(12 / 16)$ & $63(10 / 16)$ & $56(9 / 16)$ & $44(7 / 16)$ & $59^{0.02}(38 / 64)$ \\
\hline
\end{tabular}

The Avg column simply adds up the Trust and Repay choices across the four games, giving the aggregate percentage (number) of Trust and Repay choices for each group. Superscripts are one-tailed $t$ test $p$ values between the VMPFC and the row groups, treating each individual as a single data point. Epps-Singleton tests also yielded significant differences $(p<0.05)$ between the VMPFC group and both comparison groups on the Avg Repay measure (see supplemental Results, available at www.jneurosci.org as supplemental material).

Table 2. Parameter estimates derived from the pooled dataset

\begin{tabular}{llllll}
\hline Group $(N)$ & Guilt $(\beta)$ & Envy $(\alpha)$ & Expected guilt $E(\beta)$ & Expected envy $E(\alpha)$ & Group $\lambda$ parameters \\
\hline $\operatorname{VMPFC}(6)$ & $27(22)$ & $8.5(12)$ & $98(56)$ & $472(220)$ & $0.23,0.12$ \\
BDC (17) & $75^{0.06}(132)$ & $0^{0.21}(5)$ & $270^{0.29}(32)$ & $680^{0.26}(170)$ & $0.09,0.13$ \\
NC (16) & $202^{0.01}(107)$ & $0^{0.44}(8)$ & $160^{0.38}(32)$ & $466^{0.37}(165)$ & $0.15,0.12$ \\
\hline
\end{tabular}

Median estimated parameters for guilt, envy, expected guilt, and expected envy, as well as two unique $\lambda$ values estimated for each group as a whole (guilt and envy analyses, respectively). Superscripts are one-tailed $t$ test $p$ values between the VMPFC and the row groups, and numbers in parentheses are standard errors; note that in both cases, these are based on the means rather than the reported medians. See supplemental Table 2 (available at www.jneurosci.org as supplemental material) for precision estimates for the guilt estimation procedure.

groups, reflecting the large variance in performance seen in this group. These results are consistent with our above findings that the VMPFC patients displayed less guilt, although they raise the possibility that impaired behavior on the Repay decision might result from damage to structures other than the VMPFC as well.

Table 2 shows the results of the parametric analysis for guilt and envy. As suggested by the previous literature and our results from the individual game analyses, the VMPFC patients had significantly lower $\beta$ values than the comparison groups, indicating that they were relatively insensitive to guilt. To make the $\beta$ parameter more concrete, we can estimate a "betrayal point," which is the value of the temptation payoff $T$ at which, according to the estimated $\beta$, a person would be just indifferent between choosing Repay and Betray in the trust game [in the games in which the Repay payoffs are $R=(40,40)$, the betrayal point is $T(\beta)=40+$ $(\beta / 4)]$. Pure self-interest $(\beta=0)$ would yield an inferred betrayal point of 40 (because of complete indifference to the other player's payoff). Converting the estimated $\beta$ parameters into betrayal points we get $T=47$ for the VMPFC patients, lower than either of the comparison groups ( $T=59$ for BDC, and $T=91$ for NC).

A second finding from our analysis was that the $\alpha$ values for the VMPFC group were statistically indistinguishable from the comparison groups (possibly because of low statistical power and issues with the envy estimation procedure, see supplemental Methods, available at www.jneurosci.org as supplemental material). This finding supports the idea that the VMPFC patients are not diminished across the board on all emotions, as also supported by other studies (Koenigs and Tranel, 2007), but rather show selective impairments on only certain social emotions.

The parameter estimates for the subjects' beliefs about the other players' envy and guilt parameters, $E(\alpha)$ and $E(\beta)$, yielded no significant differences between groups. This helps to rule out the possibility that VMPFC patients behave anti-socially because of unusual beliefs about the other players. Estimates of the responsiveness parameter $\lambda$ are similar across the groups and reassure us that the different groups are not simply playing more or less noisily.

\section{Discussion}

Social emotions such as guilt and envy can be either assessed from subjects' reports of their feelings or inferred from behavioral observation. Typically, VMPFC patients have poor insight into their emotions, and behavioral observation in the absence of a mathematical model makes identification and interpretation of the impaired processes problematic. Furthermore, it is important to use actual decisions since it has been shown that VMPFC patients do not seem to be impaired in hypothetical decision making (Leland and Grafman, 2005). Our battery of simple economic tasks for the first time shows quantitatively that patients with damage to the VMPFC are abnormally insensitive to guilt but suggests that they are not insensitive to envy.

There are lively debates in economics, psychology and cultural anthropology about the nature of social behaviors (and their evolutionary origins) (Wood and Grafman, 2003) and in cognitive neuroscience about the neural basis of social emotions and the role of the VMPFC (Berthoz et al., 2002; Moll et al., 2003; Takahashi et al., 2004).

There is a large literature, primarily from functional imaging, that implicates the medial prefrontal cortex in "theory-of-mind" abilities; it is activated when we infer another person's beliefs or evaluate their personality (Amodio and Frith, 2006; Saxe and Powell, 2006) or even when we think about the minds of animals (Mitchell et al., 2005). Lesion studies have pointed in particular to deficits in "affective" aspects of theory-of-mind, such as empathy, irony, and social faux pas (Shamay-Tsoory et al., 2003, 2005), suggesting that the prefrontal cortex contributes to social behavior in part through the regulation of emotions, in particular social emotions (Beer et al., 2003; Ochsner and Gross, 2005). Therefore, VMPFC patients may be better viewed as dysfunctional in their ability to regulate particular social emotions. Together with our findings, these studies suggest that social emotions, such as guilt and envy, may serve to bridge the gap between decision-making in general, and social decisions, in particular. Future behavioral studies, or those using neuroimaging, could use our guilt and envy parameter estimates to make precise predictions about performance, or regional brain activation, in other social decisionmaking scenarios. Such studies could also benefit from a larger battery of games and measures (such as eliciting beliefs) which enable more precise estimation of guilt and envy parameters.

It is also interesting to compare our findings regarding impaired guilt with reports implicating VMPFC in processing regret (Camille et al., 2004; Coricelli et al., 2005). Both guilt and regret are distinguished from more basic emotions, like sadness or anger, in that they cannot be derived solely from one's own present situation. In the case of guilt, the circumstances of another person must be considered as well; in the case of regret, foregone outcomes that might have been attained must be considered. Both of these additional circumstances are abstract and require the adoption of a point of view outside one's own present circumstance. It may be that the ventromedial prefrontal cortex contributes to 
planning for the future for the same reason that it contributes to negotiating interactions with other people: it mediates the elicitation of emotions based on outcomes that are not directly experienced, but imagined.

In conclusion, the data from our battery of economic games point to a specific role in social behavior for the ventromedial prefrontal cortex. Some of the hypotheses regarding the mechanism by which damage to the vmPFC impairs social behavior are: (1) that it grossly diminishes or abolishes all emotions; (2) that it impairs complex theory-of-mind abilities but not emotions; (3) that it specifically impairs a subset of social emotions. Our data favor number (3) and argue for a relatively specific role regarding guilt in the face of otherwise intact abilities to think about other people (theory of mind) and ability to use other emotions, such as envy (Koenigs and Tranel 2007). Whether or not feelings of guilt (as opposed to their effects on motivation and behavior) are also defective in VMPFC patients remains a challenging topic for future study.

\section{References}

Ackerly SS, Benton AL (1947) Report of a case of bilateral frontal lobe defect. Assoc Res Nerv Ment Dis 27:479-504.

Amodio DM, Frith CD (2006) Meeting of minds: the medial frontal cortex and social cognition. Nat Rev Neurosci 7:268-277.

Anderson SW, Bechara A, Damasio H, Tranel D, Damasio AR (1999) Impairment of social and moral behavior related to early damage in human prefrontal cortex. Nat Neurosci 2:1032-1037.

Anderson SW, Barrash J, Bechara A, Tranel D (2006) Impairments of emotion and real-world complex behavior following childhood- or adultonset damage to ventromedial prefrontal cortex. J Int Neuropsych Soc 12:224-235.

Bechara A, Damasio H, Tranel D, Damasio AR (1997) Deciding advantageously before knowing the advantageous strategy. Science 275:1293-1295.

Bechara A, Damasio H, Damasio AR (2000) Emotion, decision making and the orbitofrontal cortex. Cereb Cortex 10:295-307.

Beer JS, Heerey EA, Keltner D, Scabini D, Knight RT (2003) The regulatory function of self-conscious emotion: insights from patients with orbitofrontal damage. J Pers Soc Psychol 85:594-604.

Berthoz S, Armony JL, Blair RJ, Dolan RJ (2002) An fMRI study of intentional and unintentional (embarrassing) violations of social norms. Brain 125:1696-1708.

Bolton GE, Ockenfels A (2000) ERC: a theory of equity, reciprocity, and competition. Am Econ Rev 90:166-193.

Breiter HC, Aharon I, Kahneman D, Dale A, Shizgal P (2001) Functional imaging of neural responses to expectancy and experience of monetary gains and losses. Neuron 30:619-639.

Camerer CF (2003) Behavioral game theory: experiments on strategic interaction. Princeton: Princeton UP.

Camerer CF, Fehr E (2006) When does economic man dominate social interaction? Science 311:47-52.

Camille N, Coricelli G, Sallet J, Pradat-Diehl P, Duhamel JR, Sirigu A (2004) The involvement of the orbitofrontal cortex in the experience of regret. Science 304:1167-1170.

Charness G, Rabin M (2002) Understanding social preferences with simple tests. Q J Econ 117:817-869.

Coricelli G, Critchley HD, Joffily M, O’Doherty JP, Sirigu A, Dolan RJ (2005) Regret and its avoidance: a neuroimaging study of choice behavior. Nat Neurosci 8:1255-1262.

Critchley HD, Mathias CJ, Dolan RJ (2001) Neural activity in the human brain relating to uncertainty and arousal during anticipation. Neuron 29:537-545.

Damasio AR (1994) Descartes' error: emotion, reason, and the human brain. New York: Putnam.

Damasio AR (2003) Looking for Spinoza: joy, sorrow, and the feeling brain. New York: Harcourt.

Fehr E, Schmidt KM (1999) A theory of fairness, competition, and cooperation. Q J Econ 114:817-868.

Fehr E, Schmidt KM (2001) Theories of fairness and reciprocity: evidence and economic applications. In: Advances in economics and econometrics-8th World Congress (Dewatripont M, Hansen L, Turnovsky S, eds). Cambridge, UK: Cambridge UP.

Frank RJ, Damasio H, Grabowski TJ (1997) Brainvox: an interactive, multimodal visualization and analysis system for neuroanatomical imaging. Neuroimage 5:13-30.

Fuster JM (1989) The prefrontal cortex. Anatomy, physiology, and neuropsychology of the frontal lobe. New York: Raven.

Hsu M, Bhatt M, Adolphs R, Tranel D, Camerer CF (2005) Neural systems responding to degrees of uncertainty in human decision-making. Science 310:1680-1683.

Koenigs M, Tranel D (2007) Irrational economic decision-making after ventromedial prefrontal damage: evidence from the ultimatum game. J Neurosci 27:951-956.

Koenigs M, Young L, Adolphs R, Tranel D, Cushman F, Hauser M, Damasio AR (2007) Damage to the prefrontal cortex increases utilitarian moral judgements. Nature 446:908-911.

Kringelbach ML (2005) The human orbitofrontal cortex: linking reward to hedonic experience. Nat Rev Neurosci 6:691-702.

Leland JW, Grafman J (2005) Experimental tests of the somatic marker hypothesis. Games Econ Behav 52:386-409.

Luce D (1959) Individual choice behavior. New York: Wiley.

Mitchell JP, Banaji MR, Macrae CN (2005) General and specific contributions of the medial prefrontal cortex to knowledge about mental states. Neuroimage 28:757-762.

Moll J, de Oliveira-Souza R, Eslinger PJ (2003) Morals and the human brain: a working model. Neuroreport 14:299-305.

Ochsner KN, Gross JJ (2005) The cognitive control of emotions. Trends Cogn Sci 9:242-249.

Rogers RD, Owen AM, Middleton HC, Williams EJ, Pickard JD, Sahakian BJ, Robbins TW (1999) Choosing between small, likely rewards and large, unlikely rewards activates inferior and orbital prefrontal cortex. J Neurosci 19:9029-9038.

Saxe R, Powell LJ (2006) It's the thought that counts: specific brain regions for one component of theory of mind. Psychol Sci 17:692-699.

Shamay-Tsoory SG, Tomer R, Berger BD, Aharon-Peretz J (2003) Characterization of empathy deficits following prefrontal brain damage: the role of the right ventromedial prefrontal cortex. J Cogn Neurosci 15:324-337.

Shamay-Tsoory SG, Tomer R, Berger BD, Goldsher D, Aharon-Peretz J (2005) Impaired "affective theory of mind" is associated with right ventromedial prefrontal damage. Cogn Behav Neurol 18:55-67.

Shamay-Tsoory SG, Tibi-Elhanany Y, Aharon-Peretz J (2007) The greeneyed monster and malicious joy: the neuroanatomical bases of envy and gloating (schadenfreude). Brain 130:1663-1678.

Shin LM, Dougherty DD, Orr SP, Pitman RK, Lasko M, Macklin ML, Alpert NM, Fischman AJ, Rauch SL (2000) Activation of anterior paralimbic structures during guilt-related script-driven imagery. Biol Psychiat 48:43-50.

Takahashi H, Yahata N, Koeda M, Matsuda T, Asai K, Okubo Y (2004) Brain activation associated with evaluative processes of guilt and embarrassment: an fMRI study. Neuroimage 23:967-974.

Wood JN, Grafman J (2003) Human prefrontal cortex: processing and representational perspectives. Nat Rev Neurosci 4:139-147. 